\title{
Integrated anti-terrorism physics-based modelling part 1: threats, loads and structural response
}

\author{
F. A. Maestas, J. L. Smith \& L. A. Young \\ Applied Research Associates, Inc., USA
}

\begin{abstract}
Modelling of a terrorist attack in an urban environment requires a balanced understanding of the complex physical processes that occur and management of the inherent uncertainties associated with the modelling. This paper examines the key physics-based techniques required to accurately model a terrorist attack in an urban environment. Specifically this paper will address the blast and fragment environment that results from an Improvised Explosive Device (IED) detonation, the loads on an urban structure and the response of that structure, to include progressive collapse. Tools such as the United States Air Force Research Laboratory's (AFRL's) Modular Effectiveness/Vulnerability Assessment (MEVA) code and the Defense Threat Reduction Agency's (DTRA's) Integrated Modular Effectiveness Analysis (IMEA) tool embody algorithms for blast and fragment environment characterization, structural response analyses, and structural assessments. The key physics-based algorithms in these tools and others will be highlighted. Additionally this paper will provide an approach to handling the uncertainties of modelling the urban structure given limited knowledge of the building's key structural attributes. The combination of treating uncertainties in a physics-based approach provides an integrated modelling method for evaluating and planning for a terrorist attack.
\end{abstract}

Keywords: weapon effectiveness, survivability analysis, modelling and simulation, physical security analysis, personnel security.

\section{Introduction}

Modelling of a terrorist attack in an urban environment requires a balanced understanding of the complex physical processes that occur and management of 
the inherent uncertainties associated with the modelling. The process used in most modelling tools can be summarized with a simple acronym: PILR; Propagation, Interaction, Load and Response. This paper will discuss the PILR model as it applies to Improvised Explosive Devices (IED) in urban environments. The focus of the paper will be associated with blast and fragment aspects. Fig. 1 provides this concept. Propagation is the environment that results from the detonation of an IED. Interaction describes how the environment interacts with the urban structure. Load refers to the load on the structure that results from the environment. Response is how the structure and contents, to include equipment and people, respond to that environment.

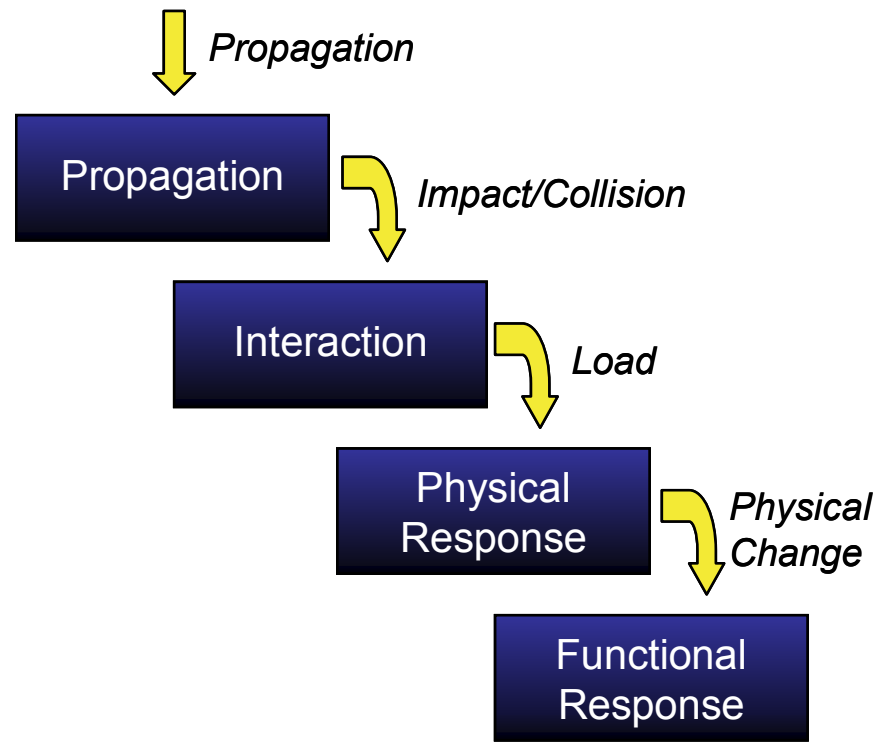

Figure 1: $\quad$ PILR representation of vulnerability modelling for facility.

\section{Propagation}

An IED detonation environment can be divided into two primary aspects, blast and fragment.

\subsection{Blast}

The level of fidelity in blast models varies somewhat from code to code. Most weapon effectiveness or survivability models provide analytical approximations for the $\operatorname{shock}(\mathrm{s})$ that result from the detonations. These blast pressure time histories for both the static (side-on) pressure and dynamic pressure environments are evaluated. Fig. 2 provides a simplified method for obtaining peak free field pressure as a function of scaled range. The scaled range is defined as the distance from the detonation to the point divided by the cube root of the 
effective explosive weight. Eqn. (1) provides a method for calculating the pressure time history. The peak pressures, time histories and the integration of the time history (impulse, fig. 3) are used as loads on the structure, equipment and inhabitants. These blast models are generally only appropriate for conventional high explosives and are used to generate the ideal, free-field environment (Needham and Crepeau [3], Kingery and Bulmash [4]).

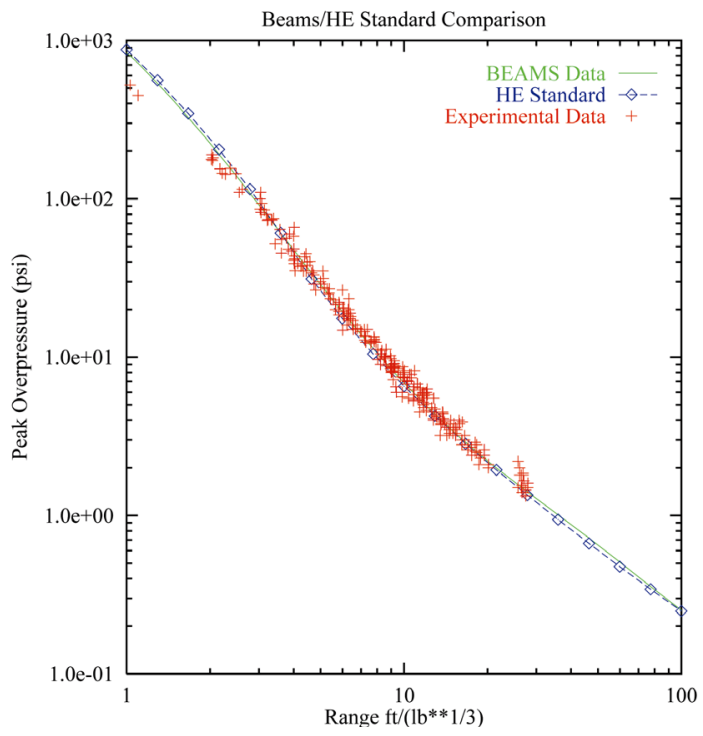

Figure 2: $\quad$ Free field peak pressure.

$$
P(t)=P_{o p}\left(1-\left(t-t_{1} / t_{o}\right)\right) e^{-\left(t-t_{1}\right) D o}
$$

where $P_{o p}$ is peak pressure, $t$ is time, $t_{1}$ is shock arrival time, $t_{o}$ is time of positive phase and $D_{o}$ is decay constant.

Fig. 4 provides the peak dynamic pressure, density, and particle velocity as a function of the peak free field pressure. Dynamic pressure, also known as "gust," is the pressure caused by motion of the gas $=1 / 2$ rho *U2, where rho is the gas density and $U$ is the gas velocity. Dynamic pressure is sometimes referred to as "differential pressure."

Since most IEDs are not made from TNT the equivalent explosive weight, $\mathrm{W}_{\mathrm{e}}$, is calculated by scaling the energy to the explosive of interest.

\subsection{Fragments}

IEDs are currently being employed by Iraqi insurgents at a rate of approximately 40 per day. Many IEDs are constructed using unexploded inventoried ordnances. Thus, fragment fly-out can be modelled using a stochastically generated set of 
weapon fragments, based upon either Arena test data files or Mott's distribution. Simplified algorithms as provided in eqns. (2)-(5) can be used. Fig. 5 provides an indication of fragment density as a function of fragment size.

$$
V=V_{G}\left[1-\left(\frac{R_{f}}{R_{o}}\right)^{2(1-\gamma)}\right]^{\frac{1}{2}}
$$

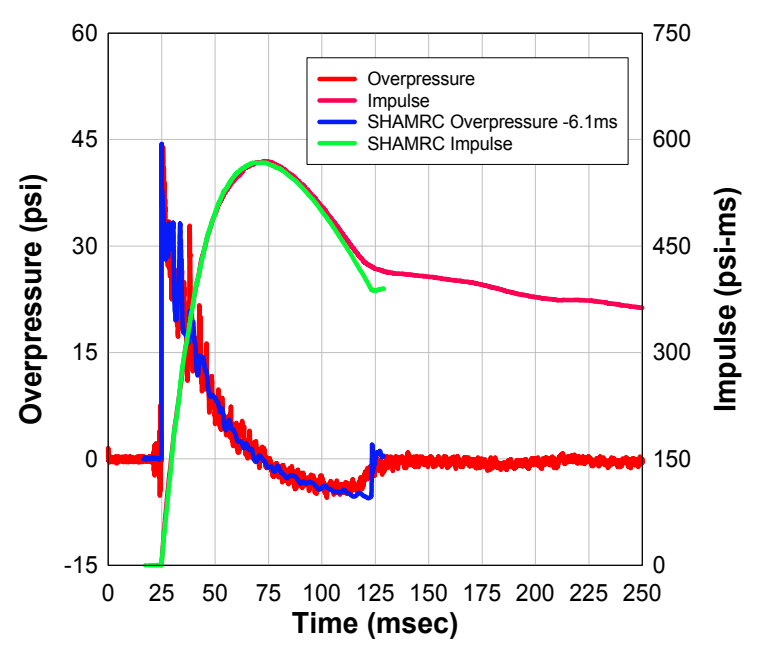

Figure 3: Example of calculated pressure and impulse time histories.

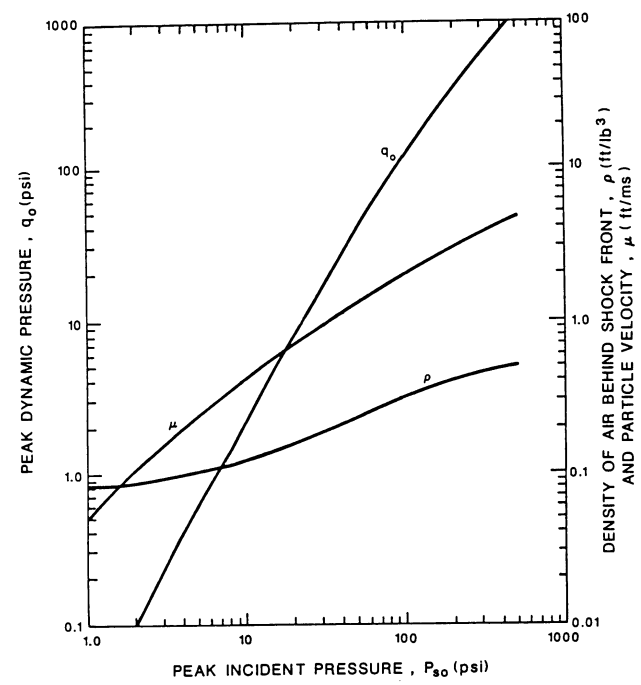

Figure 4: Peak dynamic pressure, density, and particle velocity. 


$$
V_{G}=\sqrt{2 E}\left[\frac{M}{C}+\frac{\left(1 / 2-\alpha / 3-\alpha^{2} / 6\right)}{\left(1-\alpha^{2}\right)}\right]^{\frac{1}{2}} \text { cylinder }
$$

where $M$ is mass of casing, $C$ is mass of explosive, $E$ is Gurney energy, and $\alpha=\frac{r}{R}$.

$$
\begin{aligned}
& n=\lambda e^{-\lambda y} \\
& m=y \lambda e^{-\lambda y}
\end{aligned}
$$

where $n$ is fragment density (normalized), $m$ is mass density (normalized), $N$ is total fragments (normalized), $M$ is total mass (normalized), $\lambda-\frac{1}{\text { mean fragment mass }}$, and $y$ is fragment size variable.

$$
\begin{aligned}
& N=\int_{O}^{Y} \lambda e^{-\lambda y} d y \\
& M=\int_{O}^{Y} y \lambda e^{-\lambda y} d y
\end{aligned}
$$

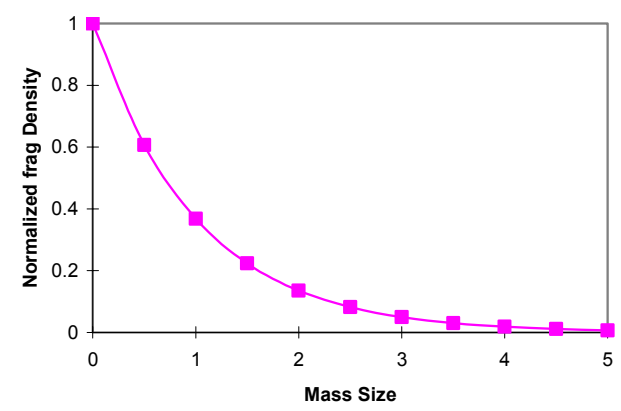

Figure 5: Example of calculated fragment size distribution interaction.

For ad hoc IED devices, fragments in a terrorist environment may be pieces of a disassembling vehicle (for a vehicle borne IED), or shards of glass or nuts and nails (for a suicide bomb). Those fragment models do not exist. The US Army Research Laboratory and others are working to address this shortfall; however, in the interim the use of Arena data from an inventory weapon would provide an upper bound of the damage caused by fragments. 


\section{Interaction}

Key to modelling the interaction of environment with the structure is accurately modelling the urban structure. This requires more than a Computer Aided Design (CAD) representation. Physical properties of the elements, such as compressive strength and their connectivity, are also required. MEVA for example, uses a 3D Complex Solid Modelling tool named the Smart Target Model Generator to add strength parameters to the facility model.

\subsection{Blast}

The free field blast environment must be adjusted based upon where the detonation occurs in relation to hard surfaces, fig. 6. For example, if the detonation occurs within a distance of $1.5 \mathrm{~W} 1 / 3$ then the peak pressure is multiplied by a factor of 1.8 . This results in an effective explosive weight, $\mathrm{W}_{\mathrm{e}}$, of $1.8 * \mathrm{~W}$. If near a corner (two surfaces) then the factor is $1.8 * 2$ (Hacker and Dunn [5]).

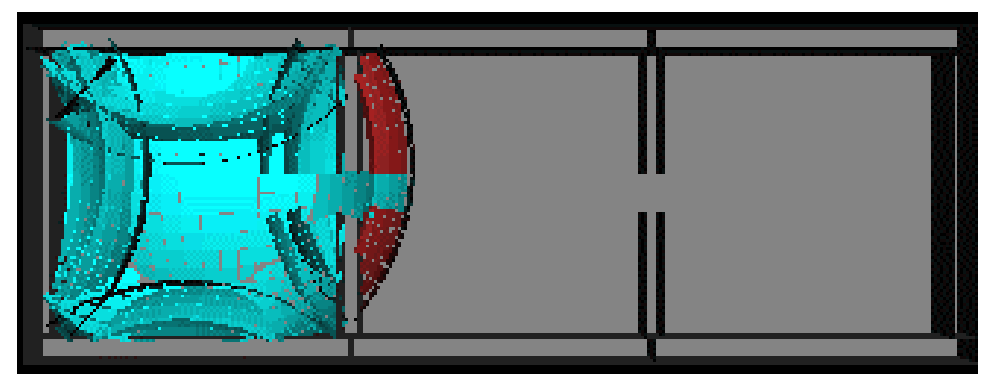

Figure 6: Example of internal blast reflection and propagation inside building.

\subsection{Fragment}

The fragment environment is typically modified by ricochet or velocity reduction because of the energy loss that occurs due to penetration. Ricochet is typically accomplished using a simple optical reflection technique provided the angle is greater than the minimum angle where perforation occurs. More detailed ricochet can be accomplished as a function of the impact surface's harness and fragment size, shape and velocity.

\section{Load}

The loads on the structural elements, equipment and inhabitants are calculated from the modified blast and fragment environments. 


\subsection{Blast}

The blast load is calculated as a function of the distance (range) from the detonation point to the component of interest. The load may be peak pressure for comparison to breach capacity or impulse for the windows. The impulse is calculated by integrating the impulse over the structural element.

\subsection{Fragment}

The fragment load is also calculated by integrating the impulse over the structural element. For equipment and personnel the load is typically momentum based.

\section{Response}

Most codes model structural response using pressure impulse techniques [8]. The damage to walls, beams, and columns are typically explicitly modelled. The loads determined from the time history approximations, modified for reflections and integrated to obtain impulse, are compared to the structural capacity of the various components of interest to determine damage. The damage is accumulated and used for evaluation of structural and personnel response. Typical structural response mechanisms are breach, shear, and flexure failure. Only flexure failure is discussed here. Window damage and structural collapse will be also discussed.

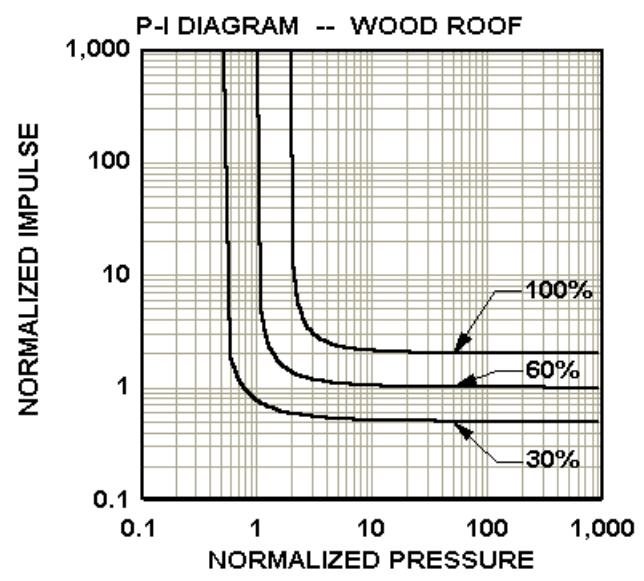

Figure 7: Example pressure impulse diagrams.

\subsection{Blast}

A simple method for addressing flexural failure is to use threshold pressureimpulse diagrams. A pressure-impulse diagram (P-I diagram) for a given structural component is a plot of the combined values of the applied pressure and impulse that lead to a given level of structural damage. That is, a P-I diagram is a 
contour curve for a given damage level that is plotted as a function of the applied pressure and impulse. As an example, P-I diagrams for a wooden roof/floor are shown in fig. 7. In these charts, the applied pressure and impulse are normalized (scaled) with respect to structural and geometric properties of the structural component. P-I diagrams derived on the basis of structural dynamics principles and available test data have been obtained [6-8] for a large variety of structural components including reinforced concrete beams, columns and slabs, wood floors and roofs, steel beams and columns, reinforced and un-reinforced masonry walls (CMU), etc. The velocity of the structural debris can be derived by making the applied impulse equal to the momentum of the debris.

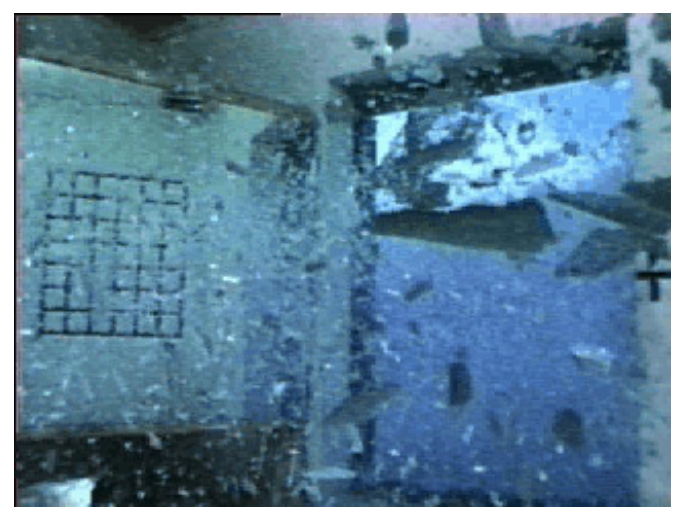

Figure 8: $\quad$ Example of glass debris.

\subsection{Windows}

Glass breakage is also typically determined based on peak pressure and total impulse. See fig. 8 for typical glass breakage. Typical glass has an average stiffness of $.8 \mathrm{psi} / \mathrm{in}$, a maximum allowed deflection of $.9 \mathrm{inch}$, an average natural frequency of $.2 \mathrm{rad} / \mathrm{sec}$ and an average natural frequency period of 30 milliseconds. Window failure occurs when the predicted dynamic deflection exceeds to the maximum allowable dynamic deflection. Eqns. (6)-(8) to determine predicted dynamic deflection are shown below. First the load duration is computed:

$$
\text { Tdur }=2 \times \text { Total impulse / peak pressure }
$$

Next, the ratio of load duration to averaged natural frequency of the equivalent SDOF:

$$
\text { TdurTn }=\text { Tdur } / T n_{-} \text {avg }
$$


The dynamic load factor (DLF) is calculated from TdurTn and a curve fit equation. The dynamic load factor is used to predict the maximum deflection of the window by multiplying the DLF by the static deflection of the window.

$$
\text { preddyn_defl }=\text { Peak pressure/average stiffness } x \text { DLF }
$$

The static value is the deflection that would occur if the peak applied pressure were applied statically to the window. Breakage is assumed to occur if the maximum predicted deflection exceeds the maximum allowable deflection.

\subsection{Collapse}

In the load transmission approach, gravity loads are determined by "trickling" the weight of each unfailed component down a tree of supports; component failures are determined by comparing loads against capacities. See fig. 9 for an example of how the collapse model re-allocates the loads. In the matrix methodology, gravity loads are determined by assembling and solving a global stiffness problem (similar to a finite element method), and component failures are determined by comparing loads in each of the assumed response modes to capacities. The mass distribution in the structure changes when structural components and connections fail. It is generally assumed in the collapse methodologies that failed components and any equipment supported by them fall onto the components below them. The component on which they fall has an additional load to support.
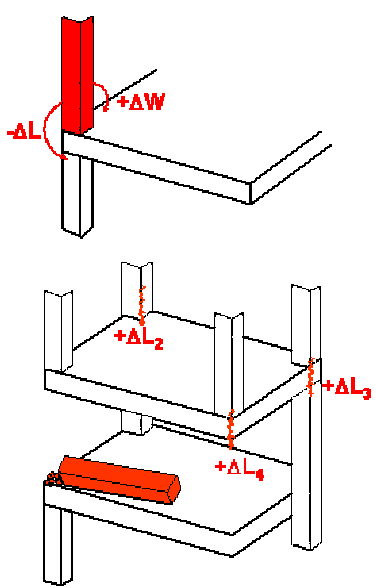

Figure 9: Example of column collapse model.

\section{Conclusion}

Modelling of a terrorist attack in an urban environment requires a balanced understanding of the complex physical processes that occur and management of the inherent uncertainties associated with the modelling. This paper provided the 
key physics-based techniques required to accurately model a terrorist attack in an urban environment. Clearly there was insufficient space to cover all the nuances of the modelling. However, the blast and fragment environment that results from an IED detonation, the loads on an urban structure and the response of that structure, including progressive collapse, were covered in a manner to allow a basic understanding of the approach.

\section{References}

[1] Young, L.A., Streit B.K., Peterson, K.J., Read, D.L. \& Maestas, F.A., Effectiveness/Vulnerability Assessments in Three Dimensions (EVA-3D) Versions 4.1F and 4.1C User's Manual - Revision A. Technical Report SL-TR-96-7000 prepared by Applied Research Associates, Inc. for U.S. Air Force Wright Laboratory, 1995.

[2] York, A.R. \& Harman, W., Integrated Munitions Effects Assessment: A Weapons Effects and Collateral Effects Assessment Tool, NBC Report, U.S. Army Nuclear and Chemical Agency, pp 30-37, Spring/Summer 2003.

[3] Needham, C.E. \& Crepeau, J.E., "The DNA Nuclear Blast Standard (1KT)," DNA 5648T, prepared by S-Cubed for the Defense Nuclear Agency, Alexandria, VA, 1981.

[4] Kingery, C.N. \& Bulmash, G., Airblast Parameters from TNT Spherical Air Bust and Hemispherical Surface Burst, Technical Report ARBRL-TR02555, U.S. Army Armament Research and Development Center, Ballistic Research Laboratory, Aberdeen Proving Ground, MD, 1984.

[5] Hacker, W.L. \& Dunn, P.E., Airblast Propagation and Damage Methodology, Final Report, AMSAA Contract No. DAAA15-94-D-0005, Delivery Order 0013, Applied Research Associates, Inc. for U.S. Army Research Laboratory, AMSRL-SL-B, Aberdeen Proving Ground, MD, 1997.

[6] Britt, J.R. \& Little, C.D. Jr., Airblast Attenuation Entranceways and Other Typical Components of Structures, Small-Scale Tests Data Report 1, Technical Report SL-81V-22, U.S. Army Engineer Waterways Experiment Station, 1984.

[7] Hikida, S. \& Needham, C.E., Low Altitude Multiple Burst (LAMB) Model, Volume I-Shock Description, DNA 5683Z-1, prepared by S-Cubed for the Defense Nuclear Agency, Alexandria, VA, 1981.

[8] Facility and Component Explosive Damage Assessment Program (FACEDAP), Theory Manual, Version 1.2, Contract No. DACA 45-91-D0019, U.S. Army Corps Engineers, Omaha, NE, 1984. 